\title{
Diseño de estrategias para mejorar la competitividad de la industria láctea
}

\author{
Designing Strategies to Improve the Competitiveness in the Dairy Industry
}

Félix Octavio Díaz ${ }^{(1)}$, John Mauricio Jiménez ${ }^{(2)}$, María Marcela Martínez ${ }^{(3)}$

${ }^{(1)}$ MSc. en Gerencia de programas sanitarios en inocuidad de alimentos. Profesor Asistente del Departamento de Ingeniería de la Universidad de Caldas. Manizales, Colombia. Grupo de Investigación en Cromatografía y Técnicas Afines. felix.diaz@ucaldas.edu.co

(2) Profesional en Ingeniería de Alimentos. Universidad de Caldas. Manizales, Colombia.jmja2411@gmail.com

(3) MSc. en Microbiología. Profesora Asistente del Departamento de Ingeniería de la Universidad de Caldas. Manizales, Colombia. Grupo de Investigación en Cromatografía y Técnicas Afines. marcela.martinez@ucaldas.edu.co

Recibido 26 de agosto de 2015. Modificado 20 de noviembre de 2015. Aprobado 27 de noviembre de 2015.

DOI: http://dx.doi.org/10.16924/riua.v0i43.865

\section{Palabras clave}

Buenas Prácticas de Ganadería, Buenas Prácticas de Manufactura, Estrategias, Industria Láctea, ISO 17025.

\section{Resumen}

La industria láctea de Manizales debe tener cambios significativos para ser más competitiva. Para dar respuesta a esta necesidad, se planteó un estudio en el que se evaluaron las condiciones fisicoquímicas y microbiológicas de la leche cruda, las Buenas Prácticas Ganaderas (BPG) y Buenas Prácticas de Ordeño (BPO) en 42 hatos lecheros. Además, se evaluó el estado de los laboratorios de las industrias lácteas según la norma ISO 17025:2005 y las condiciones de Buenas Prácticas de Manufactura (BPM) de 5 fábricas procesadoras de leche mediante una lista de chequeo. Como resultado, se obtuvo un diagnóstico y se plantearon estrategias con el fin mejorar la competitividad de la industria láctea de la ciudad.

\section{Key words}

Good Farming Practices, Dairy industry, Good Manufacturing Practices, ISO 17025, Strategies.

\begin{abstract}
The dairy industry Manizales will have to undergo significant changes in order to be more competitive. To meet this need, a study was set out to evaluate the physicochemical and microbiological conditions of raw milk, the good farming practices (GFP) and Good Milking Practices (GMP) in 42 dairy herds. In addition, the state or dairy laboratories was assessed according to ISO 17025: 2005 and GMP conditions of 5 milk-processing factories through a checklist. As a result, a diagnosis was obtained and strategies were proposed to improve the competitiveness of the dairy industry in the city.
\end{abstract}

\section{INTRODUCCIÓN}

La competitividad se refiere a la capacidad de las organizaciones para la producción de bienes y servicios de una forma eficiente, para que sean atractivos a los consumidores finales, quienes son los evaluadores principales (Orjuela, 2013).

Los problemas de la industria láctea del departamento de Caldas están relacionados con varios factores que se encuentran incluidos en la política nacional para mejorar la competitividad del sector (DNP, 2010). Dentro de los factores destacados están la disminución de los costos de producción a través de la búsqueda de alternativas alimentarias, el mejoramiento genético y la investigación e innovación tecnológica para presentar mejoras significativas y lograr la competitividad de la cadena láctea a nivel nacional. Existen otros estudios regionales (Arcila, Arcila y Ciro, 2005; Mazzeo, 2012) que han impulsado la necesidad de buscar estrategias para generar ventajas competitivas en el sector. Para ello, se han propuesto diferentes proyectos de investigación y proyección. De igual manera, la conformación de equipos idóneos de trabajo permite mejorar los aspectos más importantes en la cadena láctea, como la evaluación de las características de calidad desde el aspecto fisicoquímico y microbiológico de la leche (Ministerio de Protección Social, 2006), las buenas prácticas ganaderas (BPG) y las buenas prácticas de ordeño (BPO). Allí se hace referencia a las acciones involucradas en 
el eslabón primario de la ganadería bovina. Estas acciones van encaminadas hacia el aseguramiento de la inocuidad alimentaria, como es el caso de la leche, la protección del medio ambiente y el personal que trabaja en esta cadena (IICA, 2009).

Por otra parte, la norma ISO 17025:2005 (ICONTEC, 2005) establece las condiciones técnicas y de gestión que se deben tener en cuenta para la acreditación y certificación de un laboratorio que preste servicios. Esta clase de acreditación y certificación la aporta la Organización Nacional de Acreditación de Colombia (Organismo Nacional de Acreditación, 2010). Finalmente, las buenas prácticas de manufactura (BPM) (decreto 3075 de 1995, resolución 2674 de 2013) establece las condiciones higiénicas y sanitarias que deben cumplir todas las organizaciones que trabajan con esta clase de productos. Ellas deben ajustarse a las normatividades existentes para que las normas se cumplan de manera plena (IICA, 2009).

Teniendo en cuenta todo lo anterior, se realizó un proyecto de investigación y proyección con el objetivo de diseñar y promover estrategias para el mejoramiento de la competitividad de las industrias del sector lácteo de Manizales.

\section{Metodología}

Para el diseño de las diferentes estrategias que propiciarán el mejoramiento continuo de la competitividad de la industria láctea de Manizales, se procedió a realizar una serie de actividades de acuerdo a los siguientes objetivos propuestos: evaluar los peligros físicos, químicos y microbiológicos durante la producción de la leche de los proveedores de las industrias lácteas de Manizales (Caldas); elaborar un diagnóstico para detectar deficiencias de BPG; desarrollar estrategias para apoyar el proceso de implementación de la ISO 17025:2005 en los laboratorios del sector lácteo; e indagar sobre programas de aseguramiento de la calidad e inocuidad para las empresas procesadoras de leche. Para ello, se inició con una población de 140 fincas, de las cuales se tomaron como muestra 42, además, 5 empresas procesadoras de leche y 7 laboratorios de control de calidad. Enseguida, se tomaron muestras de leche cruda en el 2013 para realizar análisis fisicoquímicos y determinar la densidad, la acidez, el pH, los sólidos no grasos, los sólidos totales, el punto crioscópico, la proteína y la grasa. Igualmente, se hicieron análisis microbiológicos como recuento de aerobios mesófilos (RAM), termodúricos (TDR), Staphylococcus aureus, enumeración de coliformes totales y E. coli, detección de Listeria y de Salmonella, antibióticos, entre otros. Los resultados obtenidos se confrontaron con los requisitos del decreto 616 de 2006 (Ministerio de Protección Social, 2006).

Más adelante, se evaluó si las fincas productoras de leche cumplían con los programas de BPG y BPO utilizando encuestas (IICA, 2009). Así se logró determinar cuáles eran los aspectos a mejorar. Finalmente, se ejecutaron auditorías utilizando listas de chequeo, en las cuales se dieron a conocer las condiciones de los laboratorios (BPL) que existen dentro de las diferentes empresas del sector lácteo de Manizales, teniendo en cuenta la ISO 17025:2005 (Icontec, 2005) y BPM resolución 2674 de 2103 (Ministerio de Protección Social 1997; 2013).

\section{Resultados}

Los resultados de los análisis fisicoquímicos y microbiológicos de muestras de leche cruda de los proveedores de las industrias lácteas de Manizales (Caldas) se encuentran en las tablas 1 y 2. El diagnóstico para detectar deficiencias de BPG y BPO se encuentran reportados en la Tabla 3. Las estrategias para apoyar el proceso de implementación de la ISO 17025:2005 en los laboratorios del sector lácteo se encuentran en la Tabla 4. La indagación sobre programas de aseguramiento de la calidad e inocuidad (BPM) para las empresas procesadoras de leche se encuentran reportados en las tabla 6. Estos resultados fueron sometidos a tratamiento estadístico descriptivo, y para la calificación final de cada objetivo se elaboraron tablas de calificación global indicando una escala porcentual (tablas 2 y 5).

\section{Discusión}

Los resultados de la media, la desviación estándar, la varianza y el coeficiente de variación de las fincas muestreadas se encuentran en la Tabla 1. Se muestra una alta variabilidad en los diferentes atributos fisicoquímicos de la leche cruda para las fincas estudiadas. Se encontraron valores promedio cercanos a los límites de calidad establecidos en el decreto 616 de 2006 (Ministerio de Protección Social, 2006). Es importante destacar el valor por encima de los límites de norma para el porcentaje de grasa (media 3,39 \% frente a una especificación de 3,0\%). Los valores de acidez (0,17 \% de ácido láctico) y densidad (1,030 g/ml) se encuentran dentro de los límites de especificación exigidos por el decreto 616 de 2006.

La Tabla 2 muestra el promedio, la desviación estándar, la varianza y el coeficiente de variación de los resultados de los análisis microbiológicos de la leche cruda a las muestras tomadas de las diferentes fincas seleccionadas como muestra de estudio. Se encontró una desviación muy baja para los diferentes análisis realizados (RAM, TDR, S. aureus). Aunque los valores obtenidos se encuentran dentro de los límites de especificación del decreto 616 de 2006 (Ministerio de Protección Social, 2006).

Una de las estrategias propuestas para disminuir la alta variabilidad consiste en realizar controles higiénicos de la leche cruda y la contaminación con patógenos en las fincas de proveedores. Para ello, se deben implementar actividades como el diseño y la ejecución de programas que permitan reducir los riesgos de patógenos, a través de control de calidad e inocuidad de la leche cruda desde el ordeño hasta la llegada a la planta. De esta manera, se garantiza una pasteurización adecuada. 
La Tabla 3 evidencia el resumen porcentual de los resultados de los cumplimientos de BPG (IICA, 2009) por parte de los hatos encuestados indicando los programas que se manejan en mayor porcentaje de aplicabilidad. Se encuentran calificaciones bajas en variables como sanidad animal, suministro y calidad del agua. El programa condiciones de producción de leche arrojó las mejores calificaciones de cumplimiento. Con el fin de mejorar los aspectos correspondientes a las BPG y BPO se propone asumir programas adecuados como el registro y la documentación, el suministro y la calidad del agua, el control de insumos agropecuarios y de medicamentos, el manejo integrado de plagas, la sanidad animal, la determinación de volúmenes de producción de leche/hato y por vaca al día. Además, se debe profundizar en formalizar registros de los controles de la leche cruda, brindar capacitación al personal que labora en los hatos y realizar programas de limpieza adecuados para evitar la contaminación.

La Tabla 4 consigna el resumen de los valores de cumplimiento de las industrias con respecto a las buenas prácticas de laboratorio (Organismo Nacional de Acreditación, 2010). Se observa un alto cumplimiento en instalaciones sanitarias e instalaciones físicas. Un débil cumplimiento en equipos y análisis fisicoquímicos. En cambio, la Tabla 5 ilustra el promedio, la desviación estándar, la varianza y el coeficiente de variación de los resultados del cumplimiento de los laboratorios frente a una calificación global. La calificación obtenida por los laboratorios de las empresas se encuentra dentro de un rango aceptable. Con esta información, se puede inferir que las estrategias que deberían adoptar las industrias procesadoras de leche de Manizales consisten, por un lado, en promover planes de aseguramiento de la calidad analítica en sus laboratorios y, por otro, en desarrollar un sistema de gestión de calidad que siga los parámetros establecidos por la NTC/ ISO/IEC 17025:2005. Así mismo, dentro de las actividades sugeridas están la realización de capacitaciones a los operarios de los laboratorios llevando a cabo registros, la creación de acciones correctivas en las diferentes áreas del laboratorio y la realización de auditorías para verificar los cumplimientos a lo pactado. La Tabla 6 evidencia los valores de cumplimiento de las industrias con respecto a las buenas prácticas de manufactura según los requisitos del decreto 3075 de 2007 y la resolución 2654 de 2013 (Ministerio de Protección Social, 2006). Igualmente, se encuentran valores por debajo de los límites de especificación. Además, se observan niveles de desempeño bajos en programas como instalaciones sanitarias y documentación del sistema de inocuidad. Adicionalmente, el programa de metrología en algunas industrias no se encontró implementado. Finalmente, los valores observados indican posibilidades de mejora en la industria láctea de Manizales. Para incrementar las BPM en las industrias procesadoras, se propone la adopción total de los programas de aseguramiento de la inocuidad de los alimentos y de las normas de higiene adecuadas dentro de la planta. De igual forma, sería interesante que la industria procesadora de leche adoptara el sistema de análisis de peligros y puntos críticos de control (HACCP).

\begin{tabular}{|c|c|c|c|c|c|c|c|c|c|}
\hline $\begin{array}{l}\text { PARÁMETRO } \\
\text { ESTADÍSTICO }\end{array}$ & TEMP $\left({ }^{\circ} \mathrm{C}\right)$ & $\mathrm{pH}\left(20^{\circ} \mathrm{C}\right)$ & $\begin{array}{l}\text { ACIDEZ } \\
\text { (\% Ac. } \\
\text { Láctico) }\end{array}$ & $\begin{array}{l}\text { DENSIDAD } \\
(\mathrm{g} / \mathrm{mL})\end{array}$ & $\begin{array}{c}\text { PUNTO } \\
\text { Crioscópico } \\
\left({ }^{\circ} \mathrm{C}\right)\end{array}$ & \% Grasa & \%Proteína & \% SNG & $\begin{array}{l}\text { \% Sólidos } \\
\text { totales }\end{array}$ \\
\hline MEDIA & 5,88 & 6,70 & 0,17 & 1,030 & $-0,58$ & 3,39 & 3,44 & 9,44 & 12,86 \\
\hline $\begin{array}{l}\text { DESV. } \\
\text { ESTANDAR }\end{array}$ & 1,15 & 0,06 & 0,02 & 0,00 & 0,03 & 0,51 & 0,28 & 0,52 & 0,65 \\
\hline VARIANZA & 1,33 & 0,00 & 0,00 & 0,00 & 0,00 & 3,39 & 0,08 & 0,27 & 0,43 \\
\hline $\begin{array}{c}\text { COEF. } \\
\text { VARIACION }\end{array}$ & 19,59 & 0,86 & 12,91 & 0,28 & $-4,42$ & 14,98 & 8,21 & 5,48 & 5,09 \\
\hline
\end{tabular}

Tabla 1: Análisis estadístico descriptivo de los resultados fisicoquímicos de leche cruda en 2013. Fuente: Elaboración propia.

\begin{tabular}{c|c|c|c|}
\hline PARÁMETRO ESTADISTICO & RAM (UFC/mL) & TDR (UFC/mL) & S. Aureus (UFC/mL) \\
\hline PROMEDIO & $5,9 \mathrm{E}+11$ & $3, \mathrm{E}+07$ & $6, \mathrm{E}+06$ \\
\hline DESV. ESTANDAR & $3,56671 \mathrm{E}+12$ & 175695350,5 & 36175481,12 \\
\hline VARIANZA & $1,27214 \mathrm{E}+25$ & $3,08689 \mathrm{E}+16$ & $1,30867 \mathrm{E}+15$ \\
\hline COEF. VARIACION & 600,00 & 599,96 & 599,43 \\
\hline
\end{tabular}

Tabla 2: Análisis estadístico descriptivo de los resultados microbiológicos de leche cruda en 2013. Fuente: Elaboración propia. 


\begin{tabular}{|c|c|}
\hline Factores de BPG & $\begin{array}{c}\text { Calificación promedio de } \\
\text { variables (\%) }\end{array}$ \\
\hline Sanidad animal & 49,58 \\
\hline Suministro y calidad de agua & 27,08 \\
\hline $\begin{array}{c}\text { Control de medicamentos e insumos } \\
\text { agropecuarios }\end{array}$ & 38,24 \\
\hline Instalaciones y otras áreas & 58,33 \\
\hline Registros y documentación & 39,89 \\
\hline Manejo integrado de plagas & 32,29 \\
\hline Bienestar animal & 61,11 \\
\hline Personal & 54,17 \\
\hline Condiciones de producción de leche & 80,00 \\
\hline
\end{tabular}

Tabla 3: Resumen de calificación de los cumplimientos de BPG en los hatos encuestados. Fuente: Elaboración propia.

\begin{tabular}{|c|c|c|c|c|c|c|c|c|c|c|c|c|c|c|}
\hline $\begin{array}{l}\text { Aspectos } \\
\text { Verificación }\end{array}$ & $\begin{array}{l}\text { Total } \\
\text { Cump. } \\
\text { CE }\end{array}$ & $\begin{array}{l}\% \\
\text { Cump. } \\
\text { CE }\end{array}$ & $\begin{array}{c}\text { Total } \\
\text { Cump. } \\
\text { NM }\end{array}$ & $\begin{array}{c}\% \\
\text { Cump } \\
\text { NM }\end{array}$ & $\begin{array}{c}\text { Total } \\
\text { Cump. } \\
\text { CL }\end{array}$ & $\begin{array}{c}\% \\
\text { Cump. } \\
\text { CL }\end{array}$ & $\begin{array}{c}\text { Total } \\
\text { Cump. } \\
\text { MA }\end{array}$ & $\begin{array}{c}\% \\
\text { Cump. } \\
\text { MA }\end{array}$ & $\begin{array}{l}\text { Tot a I } \\
\text { Cump. } \\
\text { MC }\end{array}$ & $\begin{array}{l}\% \\
\text { Cump. } \\
\text { MC }\end{array}$ & $\begin{array}{l}\text { Tot a I } \\
\text { Cump. } \\
\text { BC }\end{array}$ & $\begin{array}{l}\% \\
\text { Cump. } \\
\text { BC }\end{array}$ & $\begin{array}{l}\text { Tot a l } \\
\text { Cump. } \\
\text { UTA }\end{array}$ & $\begin{array}{c}\% \\
\text { Cump. } \\
\text { UTA }\end{array}$ \\
\hline $\begin{array}{l}\text { Instalaciones } \\
\text { Físicas }\end{array}$ & 13 & 81,25 & 12 & 75 & 13 & 81,25 & 8 & 50 & 15 & 93,75 & 13 & 81,25 & 16 & 100 \\
\hline $\begin{array}{c}\text { Instalaciones } \\
\text { Sanitarias }\end{array}$ & 3 & 100 & 3 & 100 & 3 & 100 & 3 & 100 & 3 & 100 & 1 & 33,333 & 3 & 100 \\
\hline $\begin{array}{c}\text { Recepción de } \\
\text { Muestras }\end{array}$ & 1 & 100 & 1 & 100 & 1 & 100 & 1 & 100 & 1 & 100 & 1 & 100 & 1 & 100 \\
\hline $\begin{array}{l}\text { Área Equipos } \\
\text { Microbiología }\end{array}$ & 14 & 87,50 & 11 & 68,75 & 0 & 0 & 0 & 0 & 13 & 81,25 & 14 & 87,50 & 14 & 87,50 \\
\hline $\begin{array}{c}\text { Análisis } \\
\text { Microbiológico }\end{array}$ & 8 & 61,54 & 4 & 30,77 & 3 & 0 & 3 & 23,08 & 9 & 69,23 & 12 & 92,31 & 10 & 76,92 \\
\hline $\begin{array}{l}\text { Área Equipos } \\
\text { Fisicoquímica }\end{array}$ & 21 & 56,76 & 10 & 27,03 & 10 & 8,11 & 10 & 27,03 & 7 & 45,95 & 0 & 0 & 20 & 54,05 \\
\hline $\begin{array}{c}\text { Análisis } \\
\text { Fisicoquímico }\end{array}$ & 30 & 55,56 & 14 & 52 & 16 & 29,63 & 22 & 40,74 & 16 & 29,63 & 0 & 0 & 33 & 61,11 \\
\hline $\begin{array}{l}\text { Análisis } \\
\text { Sensorial }\end{array}$ & 4 & 100 & 1 & 25 & 0 & 0 & 0 & 0 & 1 & 25 & 0 & 0 & 4 & 100 \\
\hline $\begin{array}{l}\text { Control de } \\
\text { Calidad }\end{array}$ & 9 & 90 & 7 & 70 & 5 & 50 & 6 & 60 & 7 & 70 & 8 & 80 & 8 & 80 \\
\hline $\begin{array}{c}\text { Salud } \\
\text { Ocupacional }\end{array}$ & 3 & 100 & 0 & 0 & 3 & 100 & 3 & 100 & 3 & 100 & 3 & 100 & 3 & 100 \\
\hline Saneamiento & 4 & 100 & 2 & 50 & 2 & 50 & 2 & 50 & 4 & 100 & 4 & 100 & 4 & 100 \\
\hline $\begin{array}{l}\text { Recepción y } \\
\text { Entrega de } \\
\text { Resultados }\end{array}$ & 8 & 100 & 4 & 50 & 5 & 62,5 & 7 & 87,5 & 2 & 25 & 8 & 100 & 8 & 100 \\
\hline
\end{tabular}

Tabla 4: Relación de cumplimientos y porcentajes de cumplimientos de los diferentes aspectos de verificación. Fuente: Elaboración propia. 


\begin{tabular}{|c|c|c|c|c|c|c|c|}
\hline Medida estadística & CE & NM & CL & MA & ME & BC & UTA \\
\hline Promedio & 86,05 & 54,05 & 48,46 & 53,20 & 69,98 & 64,53 & 88,30 \\
\hline Desv. Estándar & 18,08 & 30,46 & 40,76 & 37,20 & 30,97 & 42,98 & 16,70 \\
\hline Varianza & 327,03 & 927,89 & 1661,29 & 1383,83 & 959,06 & 1846,92 & 279,02 \\
\hline Coef. Variación & 21,02 & 56,36 & 84,11 & 69,93 & 44,25 & 66,60 & 18,92 \\
\hline Promedio Global del Sector & \multicolumn{1}{|c|}{$66,40 \%$} \\
\hline
\end{tabular}

Tabla 5: Análisis estadístico de los diferentes aspectos de verificación de los laboratorios de las empresas del sector lácteo de Manizales. Fuente: Elaboración propia.

\begin{tabular}{|c|c|c|c|c|c|c|c|c|c|c|c|c|c|c|c|c|c|c|c|}
\hline EMPRESAS & 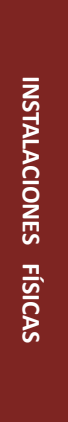 & 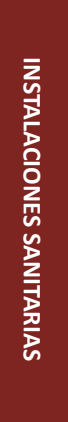 & 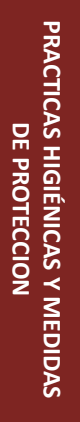 & 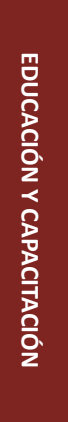 & 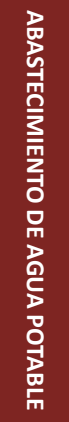 & 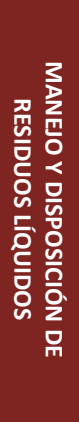 & 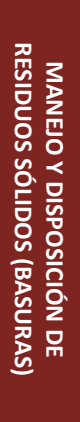 & 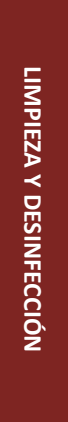 & 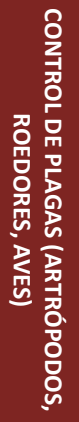 & 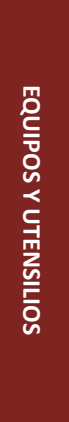 & 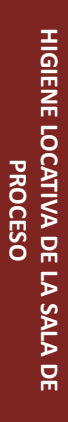 & 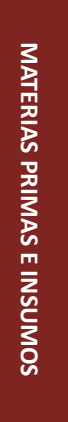 & $\begin{array}{l}\text { 塄 } \\
\text { 点 } \\
\text { 总 }\end{array}$ & 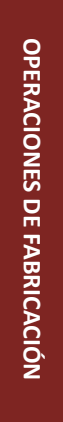 & 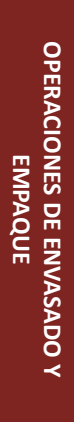 & 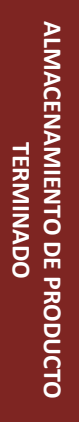 & 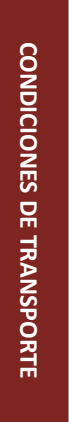 & 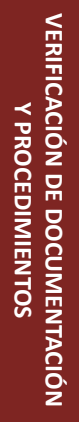 & 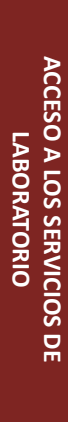 \\
\hline & $\%$ & $\%$ & $\%$ & $\%$ & $\%$ & $\%$ & $\%$ & $\%$ & $\%$ & $\%$ & $\%$ & $\%$ & $\%$ & $\%$ & $\%$ & $\%$ & $\%$ & $\%$ & $\%$ \\
\hline MC & 100 & 100 & 100 & 100 & 100 & 100 & 100 & 100 & 100 & 100 & 100 & 100 & 100 & 100 & 100 & 100 & 100 & 100 & 100 \\
\hline NM & 100 & 100 & 100 & 100 & 100 & 100 & 100 & 100 & 100 & 100 & 100 & 100 & 100 & 100 & 100 & 100 & 100 & 100 & 100 \\
\hline CE & 100 & 100 & 100 & 100 & 100 & 100 & 100 & 100 & 100 & 100 & 90 & 100 & 100 & 100 & 100 & 100 & 83 & 100 & 98 \\
\hline QEB & 79 & 60 & 92 & 50 & 38 & 100 & 40 & 50 & 80 & 75 & 95 & 60 & 100 & 75 & 67 & 83 & 83 & 0 & 72 \\
\hline MA & 100 & 80 & 100 & 100 & 78 & 100 & 100 & 100 & 100 & 94 & 100 & 100 & 100 & 100 & 100 & 100 & 100 & 100 & 97 \\
\hline $\mathrm{CL}$ & 71 & 0 & 100 & 100 & 89 & 100 & 100 & 100 & 100 & 94 & 100 & 100 & 100 & 100 & 100 & 100 & 100 & 100 & 91 \\
\hline $\begin{array}{l}\text { PROMEDIO } \\
\text { GLOBAL }\end{array}$ & 91,67 & 73,33 & 98,67 & 91,67 & 84,17 & 100,00 & 90,00 & 91,67 & 96,67 & 93,83 & 97,50 & 93,33 & 100 & 95,83 & 94,50 & 97,17 & 94,33 & 83,33 & 93,00 \\
\hline
\end{tabular}

Tabla 6: Calificación global de los parámetros de BPM de las empresas del sector lácteo de Manizales. Fuente: Elaboración propia.

\section{Conclusiones}

Primero, los análisis fisicoquímicos y microbiológicos valorados de leche cruda para el 2013 muestran que algunos aspectos se encuentran con una alta variabilidad frente a los parámetros establecidos por la correspondiente normatividad colombiana (decreto 616 de 2006). Segundo, las BPM son herramientas importantes que deben ser asumidas a la hora de realizar algún tipo de proceso industrial y agrícola. Para ello, los resultados de las auditorías de BPM en las diferentes empresas indicaron que la mayoría de las industrias se encuentran en buenas condiciones de inocuidad y cumplen favorablemente con los aspectos verificados. Tercero, los laboratorios de calidad de las diferentes empresas se encuentran en condiciones regulares respecto a las exigencias de la ISO 17025:2005 ya que, el 66,4 \% cumplen con los aspectos que se verificaron. Esto genera una incertidumbre prolongada porque no se puede determinar el verdadero estado de estos laboratorios. Cuarto, las estrategias que se deben seguir en el sector lácteo de Manizales con el fin de mejorar la competitividad comprenden: incremento en las buenas prácticas agrícolas (control de patógenos) y de manufactura (ejecución de programas de aseguramiento de la inocuidad). Adicionalmente, incremento en las competencias técnicas de los laboratorios de las industrias procesadoras (ISO 17025:2005) y mejora en la calidad de los productos. Por último, con los resultados obtenidos se observa que la competitividad de la industria láctea de Manizales se encuentra en condiciones admisibles, aunque se deben adoptar estrategias que permitan seguir mejorando. 


\section{REFERENCIAS}

Arcila, R., Aarcila, G. \& Ciro, B. (2005). Diagnóstico Regional de la cadena láctea (Caldas, Quindío, Risaralda). Manizales: Ministerio de Agricultura y Desarrollo Rural.

Departamento Nacional De Planeación (DNP). (2010). CONPES 3527. Política nacional para mejorar la competitividad del sector lácteo colombiano No. 3676. Bogotá: DNP.

Instituto Interamericano de Cooperación para la Agricultura (IICA). (2009). Buenas Prácticas de manufactura. Programa Interamericano para la Promoción del Comercio, los Negocios Agrícolas y la Inocuidad de los Alimentos. Serie de agronegocios. San José de Costa Rica: IICA.

Instituto Colombiano de Normas Técnicas y Certificación (ICONTEC). (2005). Norma NTC/ISO/IEC 17025: Requisitos Generales para la competencia de los laboratorios de ensayo y calibración.Recuperado de: http:// www.itp.gob.pe/normatividad/demos/doc/Normas\%20 Internacionales/Union\%20Europea/ISO/ISO17025LaboratorioEnsayo.pdf

Mazzeo, M. (2012). Diseño e implementación del sistema de gestión de inocuidad lácteo. Madrid: Editorial Académica Española.
Ministerio de la Protección Social (MPS). (1997). Decreto 3075 de 1997. Recuperado de: https://www.invima.gov. co/images/stories/aliementos/decreto_3075_1997.pdf

Ministerio de la Protección Social (MPS). (2006). Decreto 616 de 2006. Recuperado de: http://www.ica. gov.co/getattachment/15425e0f-81fb-4111-b21563e61e9e9130/2006D616.aspx

Ministerio de la Protección Social (MPS). (2013). Resolución 2674 de 2013. Recuperado de: http://www. ica.gov.co/getattachment/15425e0f-81fb-4111-b21563e61e9e9130/2006D616.aspx

Organismo Nacional de Acreditación (ONAC). (2010). La acreditación en Colombia. Recuperado de: http://www.onac.org.co/modulos/contenido/default. asp?idmodulo=243

ORJUELA, A. (2013). Estudio de la cadena láctea y su aporte a la competitividad de la zona Noroccidental del municipio de Pasto, departamento de Nariño. (Tesis inédita de pregrado) Universidad Nacional Abierta y a Distancia UNAD, San Juan de Pasto, Colombia. 